Bull. Austral. Math. Soc.

VoL. $61(2000) \quad$ [277-288]

\title{
ON COMPACT GROUP EXTENSION OF BERNOULLI SHIFTS
}

\author{
YOUNGHO AHN
}

Let $\rho: G \rightarrow \mathcal{U}(H)$ be an irreducible unitary representation of a compact group $G$ where $\mathcal{U}(H)$ is a set of unitary operators of finite dimensional Hilbert space $H$. For the $\left(p_{1}, \cdots, p_{L}\right)$-Bernoulli shift, the solvability of $\rho(\phi(x)) g(T x)=g(x)$ is investigated, where $\phi(x)$ is a step function.

\section{INTRODUCTION}

Let $(X, \mathcal{B}, \mu)$ be a probability space and $T$ a measure preserving transformation on $X$. A transformation $T$ on $X$ is called ergodic if the constant function is the only $T$-invariant function and it is called weakly mixing if the constant function is the only eigenfunction with respect to $T$. Let $\mathbf{1}_{E}$ be the characteristic function of a set $E$ and consider the behaviour of the sequence $\sum_{k=0}^{n-1} 1_{E}\left(T^{k} x\right)$ which equals the number of times that the points $T^{k} x$ visit $E$. The Birkhoff Ergodic Theorem applied to the ergodic transformation $T: x \mapsto\{L x\}$ on $[0,1)$, where $L$ is positive integer and $\{t\}$ is the fractional part of $t$, gives the classical Borel Theorem on normal numbers:

$$
\lim _{n \rightarrow \infty} \frac{1}{n} \sum_{k=0}^{n-1} \mathbf{1}_{((j-1) / L, j / L)}\left(T^{k} x\right)=\frac{1}{L}
$$

for $1 \leqslant j \leqslant L$. This implies that almost everywhere $x$ is $L$-normal, that is, the relative frequency of the digit $j$ in the $L$-adic expansion of $x$ is $1 / L$. See [11].

In this paper, we are interested in the uniform distribution of the sequence $d_{n} \in$ $\{0, \cdots, M-1\}$ defined by

$$
d_{n}(x) \equiv \sum_{k=0}^{n-1} \mathbf{1}_{E}\left(T^{k} x\right) \quad(\bmod M)
$$

for $T: x \mapsto\{L x\}$ and more general transformations.

\section{Received 17th June, 1999}

The author would like to thank Professor Geon Ho Choe for his advice and warm encouragement over many years. The author was supported in part by GARC-SRC.

Copyright Clearance Centre, Inc. Serial-fee code: 0004-9727/00 \$A2.00+0.00. 
DEFINITION 1: Let $T$ be a transformation on $[0,1)$ defined by

$$
T(x)=\frac{x-\sum_{k=0}^{i-1} p_{k}}{p_{i}} \quad \text { on }\left[\sum_{k=0}^{i-1} p_{k}, \sum_{k=0}^{i} p_{k}\right)
$$

where $p_{0}=0, p_{i}>0$ for $1 \leqslant i \leqslant L$ and $\sum_{k=0}^{L} p_{k}=1$. We call this transformation the $\left(p_{1}, \cdots, p_{L}\right)$-transformation.

Let $\mathcal{P}=\left\{P_{1}, \cdots P_{L}\right\}$ be a partition on $[0,1)$ with $P_{i}=\left[\sum_{k=0}^{i-1} p_{k}, \sum_{k=0}^{i} p_{k}\right)$ for $1 \leqslant i \leqslant L$. Recall that the $\left(p_{1}, \cdots, p_{L}\right)$-transformation preserves Lebesgue measure $\mu$ on $[0,1)$ and that $\mathcal{P}$ is a generating partition on $[0,1)$ with respect to the $\left(p_{1}, \cdots, p_{L}\right)$-transformation. Hence almost every $x \in[0,1)$ has a symbolic representation $\left[a_{1}, a_{2}, \cdots\right]$ with respect to the $\left(p_{1}, \cdots, p_{L}\right)$-transformation and the partition $\mathcal{P}$ where $1 \leqslant a_{i} \leqslant L$. When $x$ is represented by $\left[a_{1}, \cdots a_{n}\right]$ with a finite length, we call it a generalised $L$-adic number. Recall that a one-sided $\left(p_{1}, \cdots, p_{L}\right)$-Bernoulli shift, where $\sum_{i=1}^{L} p_{i}=1$ and $p_{i}>0$ is measure theoretically isomorphic to the $\left(p_{1}, \cdots, p_{L}\right)$-transformation on $X=[0,1)$ with Lebesgue measure $\mu$ and the partition $\mathcal{P}=\left\{P_{1}, \cdots P_{L}\right\}$.

This type of problem was first studied by Veech. He considered the case when the transformations are given by irrational rotations on the unit circle and $M=2$, and obtained results which showed that the length of the interval $E$ and the rotational angle $\theta$ are closely related. For example, he proved that when the irrational number $\theta$ has bounded partial quotients in its continued fraction expansion, the sequence $d_{n}$ is evenly distributed if the length of the interval is not an integral multiple of $\theta$ modulo 1 [10].

In [1], Ahn, Choe and Lemánczyk consider the case of the $(1 / L, \cdots ; 1 / L)$-transformation on $X=[0,1)$ and $M=2$, and show that the sequence $\left\{d_{n}\right\}$ is evenly distributed if $\exp \left(\pi i 1_{E}(x)\right)$ has finite $L$-adic discontinuity points $1 / L \leqslant t_{1}<\cdots<t_{n} \leqslant 1$. Recently, Choe, Hamachi and Nakada [2] show that $\left\{d_{n}\right\}$ is evenly distributed for more general sets and that the $\mathbb{Z}_{2}$-extension induced by $\phi(x)=\exp \left(\pi i \mathbf{1}_{B}(x)\right)$ where $\mathbf{1}_{B}$ is the characteristic function of $B$, is ergodic. In this paper, we show that for all Bernoulli shifts the sequence $\left\{d_{n}\right\}$ is uniformly distributed and that the compact group extension by $\phi(x)$ is weakly mixing. When $T$ is an irrational rotation, and $\phi(x)$ is a step function, the spectral type has been investigated by some mathematicians $[3,4,6]$. In connection with Veech's results, we also investigate the sequence $\left\{d_{n}\right\}$ induced by intervals.

To investigate the sequence $\left\{d_{n}(x)\right\}$, we consider the behaviour of the sequence $\exp \left((2 \pi i / M) d_{n}(x)\right)$ and check whether this sequence is uniformly distributed on the compact group $G$ generated by $\exp (2 \pi i / M)$. Weyl's criterion on uniform distribution 
says that the sequence $\exp \left((2 \pi i / M) d_{n}(x)\right)$ is uniformly distributed if and only if

$$
\lim _{N \rightarrow \infty} \sum_{n=1}^{N} \exp ^{k}\left(\frac{2 \pi i}{M} d_{n}(x)\right)=0
$$

for all $1 \leqslant k \leqslant L-1$.

We investigate the problem from the viewpoint of spectral theory. Let $(X, \mu)$ be a probability space and $T$ an ergodic measure preserving transformation on $X$, which is not necessarily invertible. Let $\phi(x)$ be the $G$-valued function defined by $\phi(x)=\exp \left((2 \pi i / M) \mathbf{1}_{E}(x)\right)$. Consider the skew product transformation $T_{\phi}$ on $X \times G$ defined by

$$
T_{\phi}(x, g)=(T x, \phi(x) g)
$$

Then the problem is equivalent to checking whether $T_{\phi}$ is ergodic or not.

\section{Compact group EXtension}

Let $G$ be a compact group with normalised right Haar measure $\nu$, and $(X, \mu)$ a probability space and $T: X \rightarrow X$ an ergodic measure preserving transformation. Given a function $\phi: X \rightarrow G$, define a skew product transformation $T_{\phi}: X \times G \rightarrow X \times G$ by $(x, g) \mapsto(T x, \phi(x) \cdot g)$. Then $T_{\phi}$ preserves the product measure $\mu \times \nu$. The ergodicity of $T_{\phi}$ can be checked by the decomposition of $L^{2}(X \times G)$. The Peter-Weyl Theorem says that the matrix coefficients of the irreducible unitary representation form an orthogonal basis for $L^{2}(G, \nu)$. Take any irreducible unitary representation $\rho$ and let $\left(\rho_{i j}\right)$ be its matrix representation. Then

$$
\begin{aligned}
U_{T_{\phi}}\left(\rho_{i j}(g) f(x)\right) & =\rho_{i j}(\phi(x) \cdot g) f(T x) \\
& =\sum_{k} \rho_{i k}(g) \rho_{k j}(\phi(x)) f(T x) .
\end{aligned}
$$

Hence we have the following $U_{T_{\phi}}$-invariant orthogonal decomposition:

$$
L^{2}(X \times G)=\oplus L_{\rho}^{2}(X \times G)
$$

where the subspace $L_{\rho}^{2}(X \times G)$ is spanned by functions of the form $\rho_{i j}(g) f(x), f \in L^{2}(X)$. For $\rho$ is equal to the two Hilbert spaces $L_{\rho}^{2}(X \times G)$ and $L_{\rho}^{2}(X)$ are identical. The following is a well-known fact.

LEMMA 1.

(i) The skew product transformation $T_{\phi}: X \times G \rightarrow X \times G$ is not ergodic if and only if there exists an irreducible representation $\rho \neq 1$ satisfying $\rho(\phi(x)) h(T x)=h(x)$ for some nonzero $h=\left(h_{i}\right)_{1 \leqslant i \leqslant d}, h_{i} \in L^{2}(X)$ where $d$ is the dimension of $\rho$. 
(ii) $T_{\phi}$ is not weakly mixing if and only if there exists an irreducible representation $\rho \neq 1$ and some constant $\lambda \in \mathbb{C},|\lambda|=1$, satisfying $\rho(\phi(x)) h(T x)=$ $\lambda h(x)$. Here, $h=\left(h_{i}\right)_{1 \leqslant i \leqslant d}, h_{i} \in L^{2}(X)$ is non zero and $d$ is the dimension of $\rho$.

From now on, let $H$ be a finite dimensional Hilbert space and $\mathcal{U}(H)$ be a set of unitary operators on $H$.

LEMMA 2. Let $f(x)$ be a $\mathcal{U}(H)$-valued step function with finitely many points of discontinuity. For the $\left(p_{1}, \cdots, p_{L}\right)$-transformation $T$, if an $H$-valued function $h(x)$ satisfies the equation $f(x) h(T x)=h(x)$, then $h(x)$ is also a step function with finitely many points of discontinuity.

Proof: Since $f(x) \in \mathcal{U}(H)$ and $T$ is an ergodic transformation, we may assume that $\|h(x)\|_{H}=1$ where $\|\cdot\|_{H}$ is the Hilbert space norm.

For simplicity of proof we shall prove the theorem for the transformation defined by $(p, q)$ where $p \geqslant q$. Let $\mathcal{P}$ be a partition and $\mathcal{P}_{N}=\bigvee_{k=0}^{N-1} T^{-k} \mathcal{P}$. Let $m$ be the cardinality of the set of discontinuities $Y$ and $Y_{\varepsilon}$ be an $\varepsilon$-neighbourhood of $Y$. Then there exists $\varepsilon_{0}$ such that for all $0<\varepsilon<\varepsilon_{0}, \mu\left(Y_{\varepsilon}\right)=2 m \varepsilon$. Now choose an integer $N$ such that $p^{N}<\varepsilon_{0}$ and $\left(2 m \cdot p^{N+1}\right) /(1-p)<1 / 2$.

If $I \in \mathcal{P}_{N}$ and if $I \cap Y \neq \emptyset$, then $I \subset Y_{\varepsilon}$ for $\varepsilon=p^{N}$. Hence the totality of $I \in \mathcal{P}_{N}$ with $I \cap Y \neq \emptyset$ has measure at most $2 m \cdot p^{N}$. By a similar argument, the totality of $I \in \mathcal{P}_{N+j}, j \geqslant 0$ such that $I \cap Y \neq \emptyset$ has measure at most $2 m \cdot p^{N+j}$.

Fix $L>0$ and consider the collection of $I \in \mathcal{P}_{N+L}$ having the property that $T^{j} I \cap Y \neq$ $\emptyset$ for some $0 \leqslant j \leqslant L-1$. Since $T^{j} \in \mathcal{P}_{N+L-j}$ for these $j$, and $T$ is measure preserving, these intervals have total measure at most

$$
2 m \cdot p^{N+L-1}+2 m \cdot p^{N+L-2} \cdots 2 m \cdot p^{N+1} \leqslant \frac{2 m \cdot p^{N+1}}{1-p} \leqslant \frac{1}{2} .
$$

Let $Q(N, L)$ be the sub collection of $\mathcal{P}_{N+L}$ such that $T^{j} I \cap Y=\emptyset$ for all $0 \leqslant j \leqslant L-1$. Then for each $I \in Q(N, L)$

$$
f(x) f(T x) \cdots f\left(T^{L-1} x\right)
$$

is constant, say $\Lambda(I, L) \in \mathcal{U}(H)$. Since $h(x)=f(x) h(T x)$,

$$
h(x)=f(x) f(T x) \cdots f\left(T^{L-1} x\right) h\left(T^{L} x\right) .
$$

Hence $h(x)=\Lambda(I, L) h\left(T^{L} x\right)$ holds almost everywhere on $I$. Letting $T^{L} I=J \in \mathcal{P}_{N}$, the map $T^{L}: I \rightarrow J$ is bijective and it is easily shown that

$$
\frac{1}{\mu(I)} \int_{I} h(x) d \mu(x)=\Lambda(I, L)\left(\frac{1}{\mu(J)} \int_{J} h(y) d \mu(y)\right) .
$$

Since $Q(N, L)$ measures at least $1 / 2$, the set of $x$ which is interior to some $I \in$ $Q(N, L)$ for an infinitely number of $L$ must also measure at least $1 / 2$. Fixing such an 
$x$, we have that (1) holds. We may assume that $x$ is a Lebesgue point of $h$. Since $\mathcal{P}_{N}$ is finite, it can be assumed that $J$ is always the same on the right side of (1). By the Lebesgue density theorem [8], we can assume that the left side of (1) tends to $h(x)$. By the compactness of $\mathcal{U}(H)$, we may assume that $\lim _{L \rightarrow \infty} \Lambda(I, L)=\Lambda \in \mathcal{U}(H)$. Hence

$$
h(x)=\Lambda\left(\frac{1}{\mu(J)} \int_{J} h(y) d \mu(y)\right)
$$

Since $\|h(x)\|_{H}=1$ almost everywhere, we may assume that $\|h(x)\|_{H}=1$. Since $\Lambda \in \mathcal{U}(H)$

$$
\left\|\frac{1}{\mu(J)} \int_{J} h(y) d \mu(y)\right\|_{H}=1 .
$$

$\|h(x)\|_{H}=1$ almost everywhere implies $h$ is constant on $J$.

Since $f(x)$ is a $\mathcal{U}(H)$-valued step function with finitely many discontinuities and $T^{N} J=X, h(x)$ is also step function with finitely many discontinuities.

LEMMA 3. Let $\rho: G \rightarrow \mathcal{U}(H)$ be a unitary representation of the compact group $G$ by unitary operators on a Hilbert space $H$, different from the zero representation. The following properties are equivalent:

(i) $\rho$ is irreducible;

(ii) for every nonzero vector $h \in H$, the closed linear subspace generated by $\{\rho(g) h: g \in G\}$ is $H$;

(iii) the only bounded operators on $H$ commuting with all $\rho(g)(g \in G)$ are of the form $\alpha I$ where $\alpha \in \mathbb{C}$ and $I$ is the identity operator.

Proof: For the proof, see Hewitt and Ross's Book [5].

TheOREm 1. Let $G$ be a compact group, $H$ be a finite dimensional Hilbert space and $\mathcal{U}(H)$ be a set of unitary operators on $H$. Let $\rho: G \rightarrow \mathcal{U}(H)$ be a non trivial irreducible representation of $G$. Let $T$ be the $\left(p_{1}, \cdots, p_{L}\right)$-transformation. Then $\rho(\phi(x)) h(T x)=h(x)$ has no solution if $\phi(x)$ is a step function with discontinuities at $p_{1} \leqslant t_{1}<\cdots<t_{n}=1$ and the range of $\phi(x)$ is not contained in any closed proper subgroup of $G$.

ProOF: Since $\rho \neq 1$ is an irreducible representation of $G$, it is sufficient to prove that $h(x)$ is constant by Lemma 3. Letting $\rho(\phi(x))=f(x), h(x)$ is a $H$-valued step function with finite discontinuity points by Lemma 2 . Hence there exists $0<r<p_{1}$ such that $h(x)=c$ on $[0, r)$. Hence $f(x) h(x)=h(x)$ on $[0, r)$. Since $f(x)$ is a unitary operator which is constant on $\left[0, p_{1}\right)$, the conclusion follows.

REMARK 1. Let $G$ be a compact group. If $\phi(x)$ satisfies the condition of Theorem 1 , then the skew product transformation is weakly mixing. Indeed if $\rho(\phi(x)) h(T x)=\lambda h(x)$ where $\lambda \in \mathbb{C}$ and $|\lambda|=1$, then by a similar argument to that of Lemma 2, we can 
show that $h(x)$ is also step function with finitely many points of discontinuity. By the irreducible property of $\rho$ and Lemma 3, the conclusion follows.

Let $(Y, \mathcal{C}, \mu)$ be a probability space, $f \in L^{1}(Y, \mathcal{C}, \mu)$ and $\mathcal{B} \subset \mathcal{C}$ a sub $\sigma$-algebra. Put $\nu(B)=\int_{B} f d \mu$ for $B \in \mathcal{B}$. The Radon-Nikodym Theorem implies that there is a function $h \in L^{1}(Y, \mathcal{B}, \mu)$ such that $\nu(B)=\int_{B} h d \mu$ for $B \in \mathcal{B}$. We use the notation $E(f \mid \mathcal{B})$ for $h$, and call it the conditional expectation of $f$ with respect to $\mathcal{B}$. Let $S$ be a transformation defined on $Y$ and $\mathcal{B}$ be exhaustive that is, $S^{-1} \mathcal{B} \subset \mathcal{B}$ and $S^{n} \mathcal{B} \uparrow \mathcal{C}$. The Martingale Theorem says that $E\left(f \mid S^{n} \mathcal{B}\right)$ converges to $f$ almost everywhere and in $L^{1}(Y, \mathcal{C}, \mu)$ for $f \in L^{1}(Y, \mathcal{C}, \mu)$

LEMMA 4. Let $S$ be a transformation on $(Y, \mathcal{C}, \mu)$, and $\mathcal{B} \subset \mathcal{C}$ be an exhaustive sub $\sigma$-algebra, and let $\phi: Y \rightarrow \mathcal{U}(H)$ be a $\mathcal{B}$-measurable. If $q: Y \rightarrow H$ is a $\mathcal{C}$-measurable solution to the equation $\phi \cdot q=q \circ S$, then $q$ is $\mathcal{B}$-measurable.

PROOF: We follow an idea of Parry [7]. Applying the conditional expectation operator $E(\cdot \mid \mathcal{B})$ to the equation

$$
\phi \cdot q=q \circ S
$$

we have

$$
\phi \cdot E(q \mid \mathcal{B})=E(q \circ S \mid \mathcal{B})
$$

or

$$
\phi \cdot E(q \mid \mathcal{B})=E(q \mid S \mathcal{B}) \circ S .
$$

Multiplying (2) by the Hermitian conjugate of (1) we obtain

$$
q^{*}(y) \cdot E(q \mid \mathcal{B})(y)=q^{*}(S y) \cdot E(q \mid S \mathcal{B}) \circ S(y) \quad \text { almost everywhere }
$$

where $q^{*}$ is the conjugate of $q$.

Hence

$$
\int_{Y} q^{*} \cdot E(q \mid \mathcal{B}) d \mu=\int_{Y} q^{*} \cdot E(q \mid S \mathcal{B}) d \mu .
$$

By exactly the same argument, using $S^{n} \mathcal{B}$ in place of $\mathcal{B}$, we have

$$
\int_{Y} q^{*} \cdot E\left(q \mid S^{n} \mathcal{B}\right) d \mu=\int_{Y} q^{*} \cdot E\left(q \mid S^{n+1} \mathcal{B}\right) d \mu
$$

so that

$$
\int_{Y} q^{*} \cdot E(q \mid \mathcal{B}) d \mu=\int_{Y} q^{*} \cdot E\left(q \mid S^{n} \mathcal{B}\right) d \mu
$$

Taking limits, and using the Martingale Theorem, we get

$$
\int_{Y} q^{*} \cdot E(q \mid \mathcal{B}) d \mu=\int_{Y}\|q\|_{H}^{2} d \mu
$$

where $\|\cdot\|_{H}$ is the Hilbert space norm. Thus $E(q \mid \mathcal{B})=q$ almost everywhere, and $q$ is $\mathcal{B}$-measurable. 
REMARK 2. For the $\left(p_{1}, \cdots, p_{L}\right)$-transformation and $\phi(x)$ which satisfies the condition of Theorem 1, consider the corresponding two-sided $\left(p_{1}, \cdots, p_{L}\right)$-Bernoulli transformation and the skew product transformation. Then by Lemma 4, and Remark 1, this skewproduct is weakly mixing. Hence if $G$ is metrisable, it is also Bernoulli by Rudolph's Theorem [9].

\section{MOD $M$ NORMALITY OF BERNOULLI SHIFTS}

To investigate the $\bmod M$ normality of the $\left(p_{1}, \cdots, p_{L}\right)$-transformation, we consider the function $\phi(x)=\exp \left((2 \pi i / M) \mathbf{1}_{E}(x)\right)$. Recall that a function $f(x)$ is called a coboundary if $f(x) q(T x)=q(x),|q(x)|=1$ almost everywhere on $X$. In the following two Lemmas, we consider more general functions $\phi(x)$ with finitely many discontinuity points. In the following, the unit circle in the complex plane is denoted by $\mathbb{T}$.

Lemma 5. For the $\left(p_{1}, \cdots, p_{L}\right)$-transformation, if a $\mathbb{T}$-valued function $\phi(x)$ is a step function with finitely many discontinuity points $p_{1} \leqslant t_{1}<\cdots<t_{n}<1$, then $\phi(x)$ is not a coboundary.

Proof: Assume that $\phi(x) h(T x)=h(x)$. Since $\phi(x)$ is step function with finitely many discontinuity points, $h(x)$ is also a step function with finitely many discontinuity points. Hence there exists $0<r \leqslant p_{1}$ such that $h(x)$ is constant on $[0, r)$. Thus $\phi(x) h(x)=h(x)$ on $[0, r)$. So $h(x)$ is constant on $[0,1)$. Hence the conclusion follows.

EXAMPLE 1. For the $(1 / 2,1 / 2)$-transformation, let $I=[3 / 4,1], F=\bigcup_{k=0}^{\infty}\left(1 / 2^{k}\right) I$ and $E=F \Delta T^{-1} F$. Then $\phi(x)=\exp \left(\pi i \mathbf{1}_{E}(x)\right)$ is a coboundary even if the discontinuity points of $\phi(x)$ are contained in $[1 / 2,1)$ where the cobounding function is $h(x)=\exp \left(\pi i \mathbf{1}_{F}(x)\right)$.

Now let $F=\bigcup_{k=1}^{\infty}\left(1 / 2^{k}\right) I$ and $E=F \Delta T^{-1} F$. Then $\phi(x)=\exp \left(\pi i \mathbf{1}_{E}(x)\right)$ is a coboundary even if there exists $r>0$ such that $\phi(x) \neq 1$ on $[r, 1)$. But this phenomenon disappears when $\phi(x)$ has finitely many discontinuity points. Hence we have the following Lemma.

Lemma 6. Let $\phi(x)$ be a $\mathbb{T}$-valued step function on $X=[0,1)$ with finitely many discontinuity points. If there exists $r>0$ such that $\phi(x) \neq 1$ on $[0, r)$ or $[r, 1)$, then $\phi(x)$ is not a coboundary for the $\left(p_{1}, \cdots, p_{L}\right)$-transformation.

Proof: Assume that $\phi(x) h(T x)=h(x)$. As in the proof of Lemma 5, there exists $0<r<p_{1}$ such that $h(x)$ is constant on $[0, r)$. Hence there exists $t>0$ such that $\phi(x)=1$ on $[0, t)$.

PROPOSITION 1. For the $\left(p_{1}, \cdots, p_{L}\right)$-transformation, a complex-valued function $\phi(x)=\exp \left((2 \pi i / M) 1_{(a, b)}(x)\right)$ is a coboundary if and only if $L=2, M=2$ and $(a, b)=\left(p_{1}^{2}, p_{2} p_{1}+p_{1}\right)$ or $(a, b)=\left(p_{1}^{3} /\left(1-p_{1}+p_{1}^{2}\right),\left(p_{1}^{3}-2 p_{1}^{2}+2 p_{1}\right) /\left(1-p_{1}+p_{1}^{2}\right)\right)$. 
Proof: We may assume that $0<a<b<1$ by Lemma 6 . Assume that $\phi(x) h(T x)=h(x)$. Since $\phi^{L}(x)=1, \phi^{L}(x) h^{L}(T x)=h^{L}(x)$ is equivalent to $h^{L}(T x)=$ $h^{L}(x)$. Since $T$ is ergodic, $h^{L}(x)$ is constant. Hence we may assume that $h^{L}(x)=1$. By this fact and by Lemma $3, h(x)$ can be expressed as

$$
h(x)=\exp \left(\frac{2 \pi i}{M} \sum_{k=1}^{n-1} b_{k} 1_{\left[a_{k}, a_{k+1}\right]}(x)\right)
$$

where $b_{k}$ is an integer and $0=a_{1}<a_{2}<\cdots<a_{n}=1$. We already know that if $f(x)=\lambda h(x)$, then $\phi(x) f(T x)=f(x)$ also holds. Hence we may also assume that $b_{1}=1$ and $b_{2}=0$.

Since $h(x)$ has $n-2$ discontinuity points and $h(T x)$ has at least $L(n-2)$ discontinuity points, $h(x) \overline{h(T x)}$ has at most $(L-1)(n-2)$ discontinuity points. Since $\phi(x)$ has two discontinuity points, we have

$$
0 \leqslant n-2 \leqslant \frac{2}{L-1}
$$

Hence if $L \geqslant 4$, then $\phi(x)$ can not be a coboundary. Thus the remaining case is $L=2,3$. If $L=2$, then $n=3,4$ and if $L=3$, then $n=3$.

In the following, we write by $\beta=\exp (2 \pi i / M)$ for convenience.

CASE I. Assume that $L=2$ and $n=3$. In this case, we may assume that $h(x)=\beta$ on $[0, c)$ and $h(x)=1$ on $[c, 1)$.

If $c \leqslant p_{1}$, then $\phi(x)=1$ on $\left[0, p_{1} c\right), \phi(x)=\beta$ on $\left[p_{1} c, c\right), \phi(x)=1$ on $\left[c, p_{1}\right), \phi(x)=\bar{\beta}$ on $\left[p_{1},\left(1-p_{1}\right) c+p_{1}\right)$ and $\phi(x)=1$ on $\left[0, p_{1} c\right)$. Hence $\phi(x) h(T x) \neq h(x)$.

If $c>p_{1}$, then $\phi(x)=1$ on $\left[0, p_{1} c\right), \phi(x)=\beta$ on $\left[p_{1} c, p_{1}\right), \phi(x)=1$ on $\left[p_{1}, c\right), \phi(x)=$ $\bar{\beta}$ on $\left[c,\left(1-p_{1}\right) c+p_{1}\right)$, and $\phi(x)=1$ on $\left[\left(1-p_{1}\right) c+p_{1}, 1\right)$. Hence $\phi(x) h(T x) \neq h(x)$.

If $c=p_{1}$, then $\phi(x)=1$ on $\left[0, p_{1}^{2}\right), \phi(x)=\beta$ on $\left[p_{1}^{2}, p_{1}\right), \phi(x)=\bar{\beta}$ on $\left[p_{1},\left(1-p_{1}\right) p_{1}+\right.$ $\left.p_{1}\right)$, and $\phi(x)=1$ on $\left[\left(1-p_{1}\right) p_{1}+p_{1}, 1\right)$.

Therefore

$$
\beta^{2}=1
$$

and

$$
(a, b)=\left(p_{1}^{2},\left(1-p_{1}\right) p_{1}+p_{1}\right)
$$

CASE II. Assume that $L=2$ and $n=4$. In this case, we may assume that $h(x)=\beta$ on $[0, c), h(x)=1$ on $[c, d)$ and $h(x)=\gamma$ on $[d, 1)$ where $\gamma \neq 1$. Indeed, there exists $s>p_{1} c$ and $t<\left(1-p_{1}\right) d+p_{1}$ such that $\phi(x)=1$ on $\left[0, p_{1} c\right), \phi(x)=\beta$ on $\left[p_{1} c, s\right), \phi(x)=\gamma$ on $\left[t,\left(1-p_{1}\right) d+p_{1}\right)$, and $\phi(x)=1$ on $\left[\left(1-p_{1}\right) d+p_{1}, 1\right)$. Hence $\beta=\gamma$.

If $p_{1} d>c$, then there exists $t<\left(1-p_{1}\right) d+p_{1}$ such that $\phi(x)=1$ on $\left[0, p_{1} c\right)$, $\phi(x)=\beta$ on $\left[p_{1} c, c\right), \phi(x)=1$ on $\left[c p_{1} d\right), \phi(x)=\beta$ on $\left[t,\left(1-p_{1}\right) d+p_{1}\right)$ and $\phi(x)=1$ on $\left[\left(1-p_{1}\right) d+p_{1}, 1\right)$. Hence $p_{1} d \leqslant c$. 
If $p_{1} d<c$, then there exists $t<\left(1-p_{1}\right) d+p_{1}$ such that $\phi(x)=1$ on $\left[0, p_{1} c\right)$, $\phi(x)=\beta$ on $\left[p_{1} c, p_{1} d\right), \phi(x)=1$ on $\left[p_{1} d, c\right), \phi(x)=\beta$ on $\left[t,\left(1-p_{1}\right) d+p_{1}\right)$ and $\phi(x)=1$ on $\left[\left(1-p_{1}\right) d+p_{1}, 1\right)$.

Thus $p_{1} d \leqslant c$ and by a similar argument, we can show that $\left(1-p_{1}\right) c+p=d$. Therefore $c=p_{1}^{2} /\left(1-p_{1}+p_{1}^{2}\right)$ and $d=p_{1} /\left(1-p_{1}+p_{1}^{2}\right)$. In this case, $\phi(x)=1$ on $\left[0, p_{1}^{3} /\left(1-p_{1}+p_{1}^{2}\right)\right), \phi(x)=\beta$ on $\left[p_{1}^{3} /\left(1-p_{1}+p_{1}^{2}\right), p_{1}^{2} /\left(1-p_{1}+p_{1}^{2}\right)\right), \phi(x)=\bar{\beta}$ on $\left[p_{1}^{2} /\left(1-p_{1}+p_{1}^{2}\right), p_{1} /\left(1-p_{1}+p_{1}^{2}\right)\right), \phi(x)=\beta$ on $\left[p_{1} /\left(1-p_{1}+p_{1}^{2}\right),\left(p_{1}^{3}-2 p_{1}^{2}+2 p_{1}\right) /\left(1-p_{1}+p_{1}^{2}\right)\right)$ and $\phi(x)=1$ on $\left[\left(p_{1}^{3}-2 p_{1}^{2}+2 p_{1}\right) /\left(1-p_{1}+p_{1}^{2}\right), 1\right)$.

Hence

$$
\beta^{2}=1
$$

and

$$
(a, b)=\left(\frac{p_{1}^{3}}{1-p_{1}+p_{1}^{2}}, \frac{p_{1}^{3}-2 p_{1}^{2}+2 p_{1}}{1-p_{1}+p_{1}^{2}}\right) .
$$

CASE III. Assume that $L=3$ and $n=3$. In this case, we may assume that $h(x)=\beta$ on $[0, c)$ and $h(x)=1$ on $[c, 1)$.

If $c<p_{1}$, then $\phi(x)=1$ on $\left[0, p_{1} c\right), \phi(x)=\beta$ on $\left[p_{1} c, c\right), \phi(x)=1$ on $\left[c, p_{1}\right), \phi(x)=\bar{\beta}$ on $\left[p_{1}+p_{2},\left(1-p_{1}-p_{2}\right) c+p_{1}+p_{2}\right)$ and $\phi(x)=1$ on $\left[\left(1-p_{1}-p_{2}\right) c+p_{1}+p_{2}, 1\right)$. Hence $\phi(x) h(T x) \neq h(x)$. The other case is also similarly verified.

REMARK 3. By a similar argument to that of the above proof, we can show that for the $\left(p_{1}, \cdots, p_{L}\right)$-transformation, $\phi(x)=\exp \left((2 k \pi i) / M 1_{(a, b)}(x)\right)$ is a coboundary if and only if $L=2,(k / M)=1 / 2$ and $(a, b)=\left(p_{1}^{2}, p_{2} p_{1}+p_{1}\right)$ or $(a, b)=\left(p_{1}^{3} /\left(1-p_{1}+p_{1}^{2}\right),\left(p_{1}^{3}-\right.\right.$ $\left.\left.2 p_{1}^{2}+2 p_{1}\right) /\left(1-p_{1}+p_{1}^{2}\right)\right)$.

REMARK 4. Let $G$ be the subgroup of $\mathbb{T}$ generated by $\exp (2 \pi i / M), \phi(x)$ $=\exp \left((2 \pi i) / M 1_{E}(x)\right)$ be a $G$-valued function on $X=[0,1)$ and $T_{\phi}$ be the skew product transformation on $X \times G$ defined by $T_{\phi}(x, g)=(T x, \phi(x) \cdot g)$. For the $\left(p_{1}, \cdots, p_{L}\right)$ transformation, $T_{\phi}$ is weakly mixing if $\phi(x)$ has discontinuities $p_{1} \leqslant t_{1}<\cdots<t_{n}<1$ or $E$ is an interval and $L \geqslant 3$. Hence $T_{\phi}$ is Bernoulli and $\bmod M$ normality holds almost everywhere.

Proof: Let $U_{T_{\phi}}$ be an unitary operator on $L^{2}(X \times G)$. Recall that the dual group of $G$ consists of the trivial homomorphism 1 and $\gamma_{k}$ defined by $\gamma_{k}(z)=z^{k}$ for $1 \leqslant k \leqslant M-1$. Hence

$$
L^{2}(X \times G)=\bigoplus_{k=0}^{L-1} L^{2}(X) \cdot z^{k}
$$

and each $L^{2}(X) \cdot z^{k}$ is an invariant subspace of $U_{T_{\phi}}$. If $f(x, z)$ is an eigen-function with eigenvalue $\lambda$ then $f(x, z)=\sum_{k=0}^{L-1} f_{k}(x) \cdot z^{k}$ and

$$
U_{T_{\phi}} f(x, z)=\sum_{k=0}^{L-1} \phi^{k}(x) f_{k}(T x) \cdot z^{k} .
$$


Since $T$ is weakly mixing, $f_{0}(x)$ is a constant function, $\phi^{k}(x) f_{k}(T x)=\lambda f_{k}(x)$ and $\lambda^{L}=1$ by the property of $\phi(x)$. Since $\bar{\lambda} \phi^{k}(x)$ satisfies the conditions of Proposition 1 and Lemma 5 , the conclusion follows.

Now we consider the case of the $\left(p_{1}, p_{2}\right)$-transformation, $\phi(x)=\exp \left(\pi i \mathbf{1}_{E}(x)\right)$ and $E$ being an interval. To check whether $\lim _{N \rightarrow \infty} \sum_{n=1}^{N} \exp \left(\pi i d_{n}(x)\right)=0$ or not, consider the skew product transformation $T_{\phi}$ on $[0,1) \times\{-1,1\}$ defined by $T_{\phi}(x, z)=(T x, \phi(x) \cdot z)$. Then

$$
\lim _{N \rightarrow \infty} \sum_{1}^{N} \exp \left(\pi i d_{n}(x)\right) \cdot z=\lim _{N \rightarrow \infty} \sum_{1}^{N} U_{T_{\phi}} f(x, z)
$$

where $U_{T_{\phi}}$ is an isometry on $L^{2}(X \times\{-1,1\})$ induced by $T_{\phi}$ and $f(x, z)=z$. Hence if $T_{\phi}$ is ergodic, then $\lim _{N \rightarrow \infty} \sum_{1}^{N} \exp \left(\pi i d_{n}(x)\right)=0$ by an application of the Birkhoff Ergodic theorem to $f(x, z)=z$. If $T_{\phi}$ is not ergodic, then there exists $q(x)$ such that $q(x)=\exp \left(\pi i 1_{F}(x)\right)$ for some measurable set $F$ and $\exp \left(\pi i 1_{E}(x)\right)=q(x) q(T x)$. Furthermore,

$$
\lim _{N \rightarrow \infty} \sum_{1}^{N} \exp \left(\pi i d_{n}(x)\right)=q(x) \int_{[0,1)} q(t) d \mu(t) .
$$

Hence

(i) if $(a, b)=\left(p_{1}^{2},\left(1-p_{1}\right) p_{1}+p_{1}\right)$, then

$$
\lim _{N \rightarrow \infty} \sum_{1}^{N} \exp \left(\pi i d_{n}(x)\right)=\left(2 p_{1}-1\right) \exp \left(\pi i \mathbf{1}_{(c, d)}(x)\right)
$$

where $(c, d)=\left(p_{1}, 1\right)$.

(ii) If $(a, b)=\left(p_{1}^{3} /\left(1-p_{1}+p_{1}^{2}\right),\left(p_{1}^{3}-2 p_{1}^{2}+2 p_{1}\right) /\left(1-p_{1}+p_{1}^{2}\right)\right)$, then

$$
\lim _{N \rightarrow \infty} \sum_{1}^{N} \exp \left(\pi i d_{n}(x)\right)=\left(\frac{1-3 p_{1}+3 p_{1}^{2}}{1-p_{1}+p_{1}^{2}}\right) \exp \left(\pi i \mathbf{1}_{(c, d)}(x)\right)
$$

where $(c, d)=\left(p_{1}^{2} /\left(1-p_{1}+p_{1}^{2}\right), p_{1} /\left(1-p_{1}+p_{1}^{2}\right)\right)$.

Now we consider some spectral properties of the skew product $T_{\phi}(x)$.

PROPOSITION 2. Let $T$ be an weakly mixing transformation on a probability space $(X, \mu)$ and $H_{\lambda}^{k}=\left\{h(x) \mid \phi^{k}(x) h(T x)=\lambda h(x)\right\}$ where $\phi(x)$ is a $\mathbb{T}$-valued function. Then the dimension of $H_{\lambda}^{k}$ is 0 or 1 . For each $k$, there exists at most one $\lambda$ such that the dimension of $H_{\lambda}^{k}$ is 1 .

Proof: Assume that $f(x), g(x) \in H_{\lambda}^{k}$. Then $\phi^{k}(x) f(T x)=\lambda f(x)$ and $\phi^{k}(x) g(T x)=$ $\lambda g(x)$. Hence $f(T x) \overline{g(T x)}=f(x) \overline{g(x)}$. By the ergodicity of $T, f(x) \overline{g(x)}=C$ where $C$ is constant. Thus the first assertion is proved. 
Now we shall prove the second assertion. Assume that $\phi^{k}(x) f(T x)=\lambda f(x)$ and $\phi^{k}(x) g(T x)=\lambda^{\prime} g(x)$. Hence $f(T x) \overline{g(T x)}=\lambda \cdot \lambda^{\prime} f(x) \overline{g(x)}$. By the mixing property of $T$, $f(x) \overline{g(x)}=C$ where $C$ is constant and $\lambda \cdot \lambda^{\prime}=1$.

Proposition 3. Let $T$ be an ergodic transformation on $X, G$ the finite subgroup of $\mathbb{T}$ generated by $\exp (2 \pi i / M)$ and $\phi(x)$ be a $G$-valued function. Let $T_{\phi}$ be the skew product transformation defined by $T_{\phi}(x, g)=(T x, \phi(x) \cdot g)$ on $X \times G$. If $\phi^{k}(x) h(T x)=h(x)$, then there exists $q(x)$ such that the following diagram commutes

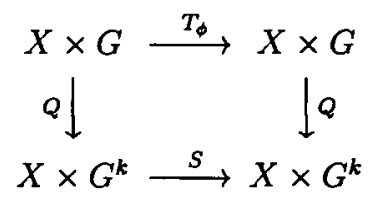

where $Q(x, g)=\left(x, q(x) g^{k}\right)$ and $S(x, g)=(T x, g)$. Hence $T_{\phi}$ has at least $r$ ergodic components where $r$ is the cardinality of $G^{k}$.

Proof: Since $\left(\phi^{k}(x)\right)^{M}(h(T x))^{M}=(h(x))^{M}$ is equivalent to $(h(T x))^{M}=(h(x))^{M}$ and $T$ is ergodic, we may assume that $(h(x))^{M}=1$. Hence there exists a $G$-valued function $q(x)$ such that $\phi^{k}(x) q(T x)=q(x)$. For this $q(x)$, it is easy to see that the diagram commutes.

EXAMPLE 2. Consider the $(1 / 2,1 / 2)$-transformation and $\phi(x)=\exp \left(\pi i \mathbf{1}_{[1 / 4,3 / 4]}(x)\right)$. Let $q(x)=\exp \left(\pi i \mathbf{1}_{[1 / 2,1]}(x)\right)$. Since $[1 / 4,3 / 4]=[1 / 2,1] \Delta T^{-1}[1 / 2,1], \phi(x)=q(x) q(T x)$. Hence $T_{\phi}$ has two ergodic components. Indeed, we can give many examples in which $T_{\phi}$ has two ergodic components: For a given $F$, let $E=F \Delta T^{-1} F, \phi(x)=\exp \left(\pi i 1_{E}(x)\right)$ and $q(x)=\exp \left(\pi i 1_{F}(x)\right)$. Then $T_{\phi}$ has two ergodic components, $\{(x, q(x)): x \in X\}$ and $\{(x,-q(x)): x \in X\}$.

\section{REFERENCES}

[1] Y. Ahn and G.H. Choe, 'Spectral types of skewed Bernoulli shift', Proc. Amer. Math. Soc. 128 (2000), 503-510.

[2] G.H. Choe, T. Hamachi and H. Nakada, 'Skew product and mod 2 normal numbers', (preprint).

[3] J.P. Conze, 'Remarques sur les transformations cylindriques et les equations fonctionnelles', Séminaire de Probabilité I (Univ. Rennes, Rennes, France 1976).

[4] J.-M. Derrien, 'Critéres d'ergodicité de cocycles en escalier. Exemples', C.R. Acad. Sci. Paris Ser. I Math. 316 (1993), 73-76.

[5] E. Hewitt and K. Ross, Abstract Harmonic analysis I, II (Springer-Verlag, Berlin, Heidelberg, New York, 1963, 1970).

[6] K.D. Merrill, 'Cohomology of step functions under irrational rotations', Israel J. Math. 52 (1985), 320-340.

[7] W. Parry, 'A Cocycle equation for shift', Contemp. Math. 135 (1992), 327-333. 
[8] W. Rudin, Real and complex analysis (McGraw-Hill, New York, 1987).

[9] D.J. Rudolph, 'Classifying the isometric extensions of a Bernoulli shift', J. Analyse Math. 34 (1978), 36-60.

[10] W.A. Veech, 'Strict ergodicity of uniform distribution and Kronecker-Weyl theorem mod 2', Tran. Amer. Math. Soc. 140 (1969), 1-33.

[11] P. Walters, An introduction to Ergodic theory (Springer-Verlag, Berlin, Heidelberg, New York, 1982).

Korea Advanced Institute of Science and Technology

Taejon 305-701

Korea

e-mail: ahn@euclid.kaist.ac.kr 